\title{
Examining the Tradeoff Between Fixed Pay and Performance-Related Pay: A Choice Experiment Approach
}

\author{
JUNYI SHEN* \\ Research Institute for Economics and Business Administration, Kobe University*
}

KAZUHITO OGAWA

Faculty of Sociology, Kansai University

HIROMASA TAKAHASHI

Faculty of International Studies, Hiroshima City University

\begin{abstract}
Previous investigations on performance-related pay have mainly analyzed its relationships with earnings, productivity, and job satisfaction. Less attention has been devoted to the investigations of individuals' preferences for the performance-related payment system per se and consequently the tradeoff between fixed pay and performance-related pay. In this paper, we first use a choice experiment approach to investigate the tradeoff between fixed pay and performance-related pay, and then link the tradeoff for each individual with their risk preferences. Our main results indicate that individuals' preferences for the payment system per se and the magnitude of tradeoffs between fixed pay and performance pay are different according to their risk preferences.
\end{abstract}

Keywords: fixed pay; performance-related pay; choice experiment; risk preference

JEL Classification: C35, J33

\section{Introduction}

Individuals are normally employed by firms under a variety of types of payment systems: fixed pay, performance-related pay, and payment combining fixed pay and performancerelated pay. The issue of effective performance and reward management has been a topic of

\footnotetext{
* Financial support from the Japanese Ministry of Education, Culture, Sports, Science and Technology through Grand-in-aid for Scientific Research (C) 25380234 is gratefully acknowledged. All of the views expressed in this paper and any errors are the sole responsibility of the authors.

Corresponding author. Junyi Shen. Research Institute for Economics and Business Administration, Kobe University, 2-1 Rokkodai, Nada, Kobe 657-8501, Japan. Tel/Fax:+ 81-78-8037013; Email: shen@rieb.kobe-u.ac.jp

(C) 2014 Junyi Shen, Kazuhito Ogawa and Hiromasa Takashi; Licensed under the Creative Commons Attribution - Noncommercial 3.0 Licence (http://creativecommons.org/licenses /bync/3.0/. Available at http://rofea.org.
} 
continuous research and discussion within the disciplines of personnel economics and human resource management (Baruch et al., 2004). In recent years, performance-related pay, either separated from or combined with fixed pay, has been much more widely used as part of the human resource policies of organizations, where the aim of this increased use has been to increase the output (e.g., number of products, quality of services, and revenues) from the input (both quantitative and qualitative) of employees.

Support for performance-related pay is theoretically grounded in expectancy theory (Pearce and Perry, 1983) and reinforcement theory (Perry et al., 2006). Expectancy theory (Vroom, 1964) is predicated on a belief that individuals will exert effort if they expect it to result in an outcome that they value. In the case of performance-related pay, employees will work harder if they value monetary rewards and believe that such rewards will result from their increased efforts. Reinforcement theory posits a direct relationship between a desired target behavior (e.g., performance) and its consequences (e.g., pay). It suggests that pay can be used to create consequences for desired behaviors such as high performance that will reinforce the behaviors (Perry et al., 2009).

Previous empirical investigations on performance-related pay have mainly analyzed its relationships with earnings (Booth and Frank, 1999; McNabb and Whitfield, 2007), with productivity (Lazear, 2000), and with job satisfaction (Green and Heywood, 2008; Heywood and Wei, 2006). Less attention has been devoted to the investigations of individuals' preferences for the performance-related payment system per se and consequently the tradeoff between fixed pay and performance-related pay. We think this may be due to it being quite difficult to find a suitable and appropriate method to elicit individuals' preferences for the tradeoff between fixed pay and performance-related pay. Therefore, the first motivation of this paper is to try to solve this preference elicitation problem by using a choice experiment approach, which is frequently applied in environmental economics, transportation economics, and marketing science. Our second purpose is to manifest the link of the magnitude of individuals' tradeoff between fixed pay and performance-related pay to their risk preferences, because this is a natural extension of the study of performance-related pay but is seldom investigated in the related literature.

Our analysis is based on data from a questionnaire survey conducted at Kansai University, Japan. In the survey, we used a choice experiment in order to elicit the tradeoff between fixed pay and performance-related pay. In the choice experiment, we do not directly observe the tradeoff, but only the respondents' choices in certain situations. In the econometric analyses, we therefore apply a latent class logit model, which assumes that the population consists of a number of latent classes and the unobserved heterogeneity among individuals can be captured by these classes through estimating a different parameter vector in the corresponding utility function. Although we do not observe the tradeoff directly, we can estimate the tradeoff values for each respondent from the parameters estimated by this model. After the tradeoff for 
each respondent has been estimated, we then examine its relationship with the risk preferences of each respondent. Note that the risk preferences of each respondent were also elicited from the questionnaire survey.

The rest of the paper is organized as follows. Section 2 contains a description of the survey and the econometric approach applied. In Section 3 we present the results from the empirical analyses, and finally in Section 4 we conclude the paper.

\section{Methodological Issues}

\subsection{Survey}

In a choice experiment, individuals are usually asked to make repeated selections of their preferred alternative in the choice sets presented to them. In our survey, before the choice sets were presented, a hypothetical scenario relating to a job description was first provided to the respondents. The scenario includes the following: (i) each respondent is assumed to undertake a 5-hour part-time job of selling snacks at a local festival; (ii) the price of each snack would be $150 \mathrm{JP}$ yen; (iii) the number of participants in the festival would be about 500; (iv) there would be no other shops selling snacks in the festival; and (v) each respondent can select their preferred payment system from the alternatives we provided.

In each choice set, we provided three alternatives, named Payment A, Payment B, and Payment C. Each payment has two common attributes - hourly pay and pay per snack sold. Note that in our experimental design, the former corresponds to fixed pay and the latter refers to performance-related pay. The attribute levels considered for each payment type are provided in Table 1. As shown in the table, since the levels of the performance-related pay in Payment A and the fixed pay in Payment B are set to be always zero, Payment A and Payment B represent a pure fixed payment system and a pure performance-related payment system, respectively. In contrast, Payment $\mathrm{C}$ is corresponds to a combined payment system with both fixed pay and performance-related pay. ${ }^{1}$

Table 1. Attributes and Their Levels for Each Payment System

\begin{tabular}{|l|c|c|c|}
\hline \multirow{2}{*}{ Attributes } & \multicolumn{3}{|c|}{ Levels of attributes } \\
\cline { 2 - 4 } & Payment A & Payment B & Payment C \\
\hline \hline Hourly pay (JP yen) & $800 / 900 / 1000$ & 0 & 500 \\
\hline Pay for each snack sold (JP yen) & 0 & $80 / 90 / 100$ & $30 / 40 / 50$ \\
\hline
\end{tabular}

\footnotetext{
${ }^{1}$ Although it is clear from the choice sets that Payments A, B, and C represent a pure fixed payment, a pure performance-related payment, and a mixed payment, we still applied these unlabeled names in order to avoid possible anchoring effects from the labels.
} 
Concerning the issue of creating the choice sets in our choice experiment, a full factorial design was adopted and 27 (i.e., $3^{3}$ ) choice sets were finally created. These choice sets were then randomly divided into 3 different blocks of choice sets, which were randomly allocated to the respondents. Each block consists of 9 choice sets. An example of a choice set is presented in Table 2.

Table 2. An Example of a Choice Set

\begin{tabular}{|l|c|c|c|}
\hline & Payment A & Payment B & Payment C \\
\hline \hline Hourly pay (JP yen) & 900 & 0 & 500 \\
\hline Pay for per snack sold (JP yen) & 0 & 100 & 40 \\
\hline $\begin{array}{l}\text { Please choose one most-desirable } \\
\text { payment plan by placing a } \checkmark \text { in a } \square\end{array}$ & $\square$ & $\square$ & $\square$ \\
\hline
\end{tabular}

The survey was conducted between July and November 2013 at Kansai University, Japan. All the respondents were undergraduates at Kansai University. They were originally recruited to attend an economic experiment. After the experiment, they were asked to answer a postexperiment questionnaire, the data of which are used for the present study. The questionnaire consists of the choice experiment questions mentioned above and several other questions related to their experimental behaviors and risk attitudes. ${ }^{2}$ The average time for answering this questionnaire was approximately 15 minutes. In total, 238 valid samples were collected, corresponding to 98 male and 140 female respondents.

\subsection{Econometric Model}

Choice models are based on random utility theory. The basic assumption embodied in the random utility approach to choice modeling is that decision makers tend to act as utility maximizers, i.e., given a set of alternatives the decision maker will choose the alternative that maximizes utility. The utility of an alternative for an individual $(U)$ is modeled as the sum of a deterministic component $(V)$ and a random error term $(\varepsilon)$. Formally, individual $q$ 's utility of alternative $i$ can be expressed as:

$$
U_{i q}=V_{i q}+\varepsilon_{i q}
$$

Hence the probability that individual $q$ chooses alternative $i$ from a particular set $J$ that comprises $j$ alternatives can be written as:

\footnotetext{
${ }^{2}$ The experimental results are to be reported in another paper.
} 
SHEN, OGAWA, TAKAHASHI Fixed Versus Performance-related Pay Tradeoff

$$
P_{i q}=P\left(U_{i q}>U_{j q} ; \forall \quad j(\neq i) \in J\right)=P\left(\varepsilon_{j q}<\varepsilon_{i q}+V_{i q}-V_{j q} ; \forall \quad j(\neq i) \in J\right) .
$$

Converting the random utility model into a choice model requires certain assumptions about the joint distribution of the vector of random error terms. If the random error terms are assumed to follow the type I extreme value (EV1) distribution and be independently and identically distributed (IID) across alternatives and cases (or observations), the multinomial (or sometimes called conditional) logit (MNL) model (McFadden, 1974) is obtained. In the MNL model, the choice probability in Equation (2) is expressed as:

$$
P_{i q}=\exp \left(\mu V_{i q}\right) / \sum_{j=1}^{J} \exp \left(\mu V_{j q}\right) .
$$

Then, making the further assumption that the deterministic component of utility is linear and additive in parameters, $V_{i q}=\beta^{\prime} X_{i q}$, the probability in Equation (3) can be given as

$$
P_{i q}=\exp \left(\mu \beta^{\prime} X_{i q}\right) / \sum_{j=1}^{J} \exp \left(\mu \beta^{\prime} X_{j q}\right)
$$

where $\mu$ represents a scale parameter that determines the scale of the utilities, which is typically normalized to 1.0 in the MNL model; $X_{i q}$ are explanatory variables of $V_{i q}$, which normally include alternative-specific constants (ASCs), the attributes of the alternative $i$, and the social-economic characteristics of the individual $q$; and $\beta^{\prime}$ is the parameter vector associated with the matrix $X_{\text {iq }}$.

It is well known that heterogeneity among individuals is extremely difficult to examine in the MNL model. This limitation could be relaxed, to some extent, by interaction terms between individual-specific characteristics and various choices. However, this method is limited in that it requires a priori selection of key individual characteristics and attributes and involves merely a limited selection of individual specific variables (Boxall and Adamowicz, 2002). One way of circumventing this difficulty is using estimation obtained from the latent class logit (LCL) model. The LCL model assumes that the population consists of a number of latent classes $S$ and the unobserved heterogeneity among individuals can be captured by these classes through estimating a different parameter vector in the corresponding utility function. Formally, the choice probability of individual $q$ of class $S$ is expressed as:

$$
P_{i q \mid s}=\exp \left(\mu_{s} \beta_{s}^{\prime} X_{i q}\right) / \sum_{j=1}^{J} \exp \left(\mu_{s} \beta_{s}^{\prime} X_{j q}\right) \quad s=1, \ldots, S
$$

where $\mu_{s}$ and $\beta_{s}^{\prime}$ are class-specific scale and utility parameters, respectively. Then, in accordance with Boxall and Adamowicz (2002), Louviere et al. (2000), and Swait (1994, 2007), the probability of individual $q$ in class $S\left(H_{q s}\right)$ can be expressed as: 


$$
H_{q s}=\exp \left(\alpha \lambda_{s}^{\prime} Z_{q}\right) / \sum_{s=1}^{S} \exp \left(\alpha \lambda_{s}^{\prime} Z_{q}\right)
$$

where $\alpha$ is a scale factor typically normalized to $1.0, \lambda_{s}^{\prime}$ is the parameter vector in class $S$, and $Z_{q}$ denotes a set of characteristics (e.g., individual-specific characteristics) determining the classification probability. Combining conditional choice equation (5) and membership classification equation (6), the unconditional probability of choosing alternative $i$ is given as:

$$
P_{i q}=\sum_{s=1}^{S} P_{i q \mid s} H_{q s}=\sum_{s=1}^{S}\left[\exp \left(\mu_{s} \beta_{s}^{\prime} X_{i q}\right) / \sum_{j=1}^{J} \exp \left(\mu_{s} \beta_{s}^{\prime} X_{j q}\right)\right]\left[\exp \left(\alpha \lambda_{s}^{\prime} Z_{q}\right) / \sum_{s=1}^{S} \exp \left(\alpha \lambda_{s}^{\prime} Z_{q}\right)\right]
$$

In equation (7), when we set $\mu_{\mathrm{s}}$ and $\alpha$ equal to one ${ }^{3}$, the parameter vectors $\beta_{s}^{\prime}$ and $\lambda_{\mathrm{s}}^{\prime}$ can be simultaneously estimated by the maximum likelihood method to explain choice behavior. ${ }^{4}$

However, the LCL model cannot be estimated unless $S$ (the number of classes) in equation (7) is given, because $S$ is discrete but maximum likelihood estimation theory requires that the parameter space be continuous and estimates be in the interior of the space (Swait, 2007). Therefore, the central issue in the LCL model is how to determine $S$. The literature has recommended a number of information criteria for this purpose (e.g., Boxall and Adamowicz, 2002; Greene and Hensher, 2003; Louviere et al., 2000; Morey el al., 2006; Shen, 2006; Swait, 2007). Among these, four measures based on the log likelihood at convergence with $s$ classes, sample size, and number of parameters are popular for determining $S$. These are defined as follows:

$$
\begin{gathered}
\text { Akaike Information Criterion, AIC }=-2\left(\log L_{s}^{*}-K_{s}\right) \\
\text { Akaike's } \rho^{2}, \bar{\rho}_{s}^{2}=1-\left[A I C_{s} /\left(-2 \cdot \log L_{0}\right)\right] \\
\text { Bozdogan Akaike Information Criterion, AIC } 3=-2 \log L_{s}^{*}+3 K_{s} \\
\text { Bayesian Information Criterion, BIC }=-\log L_{s}^{*}+\left(K_{s} \cdot \log N\right) / 2
\end{gathered}
$$

where $\log L_{s}^{*}$ is the $\log$ likelihood at convergence with $s$ classes, $K_{s}$ is the number of parameters in the model with $s$ classes, $L_{0}$ is the log likelihood of the sample with equal choice probabilities, and $N$ is the sample size.

\footnotetext{
${ }^{3}$ Boxall and Adamowicz (2002) note that utilizing the LCL model in empirical estimation requires all scale factors in equation (7) to be set equal to one.

${ }^{4}$ The parameter vector $\lambda_{s}^{\prime}$ in one of the latent classes must be normalized to zero (e.g., $\lambda_{1}^{\prime} \equiv 0$ ) to run the estimation. Therefore, the remaining $\lambda^{\prime}$ s are identified relative to this normalization.
} 
An alternative approach that can account for individual heterogeneity is called the random parameter logit (RPL) or mixed logit (ML) model; this model allows model parameters to vary randomly according to assumed distributions (e.g., normal, log-normal, or triangular) over individuals (e.g., Bhat and Gossen, 2004; Bjørner et al., 2004; Greene and Hensher, 2003; Hess et al., 2005; McFadden and Train, 2000; Revelt and Train, 1998; Train, 1998). In this approach, each individual has their own set of scale and utility parameters. From this viewpoint, one could regard the RPL/ML model as the case where each individual in the sample can be considered as an individual class, which is indeed the LCL model with $N$ (sample size) classes. In other words, the LCL model controls individual heterogeneity with $s$ classes, where $s$ is between 1 and $N$. Compared to the RPL model, there are two major advantages of the LCL model. First, the LCL approach is semi-parametric, so it does not require any specific assumptions about the distributions of parameters across individuals (Greene and Hensher, 2003). Second, the LCL model yields the probabilities in each class. This means that although each respondent is assumed to belong to one class, it is taken into account that there is uncertainty about a respondent's class membership.

\section{Results}

Tables 3 and 4 present the results associated with the MNL/LCL specifications. All the results presented were analyzed by using NLOGIT 5.0, a specialist discrete modeling package in LIMDEP (Econometric Software, Inc.). With regards to the overall impression of the MNL and LCL estimates shown in Table 4, we find that compared to the MNL model, the goodness-of-fit measures (e.g., log-likelihood, Pseudo $\mathrm{R}^{2}$, and predictive power) are significantly improved by applying the LCL approach.

Table 3. Information Criteria for Different Numbers of Latent Classes

\begin{tabular}{|c|c|c|c|c|c|}
\hline Classes & Log-likelihood & AIC & $\bar{\rho}^{2}$ & AIC3 & BIC \\
\hline \hline 1 & -2089.32 & 4186.642 & 0.104825 & 4190.642 & 2095.982 \\
\hline 2 & -1681.49 & 3380.989 & 0.277087 & 3389.989 & 1696.483 \\
\hline 3 & -1448.64 & 2925.275 & 0.374527 & 2939.275 & 1471.953 \\
\hline 4 & -1396.46 & 2830.913 & 0.394703 & 2849.913 & 1428.099 \\
\hline 5 & -1390.98 & 2829.958 & 0.394907 & 2853.958 & 1430.949 \\
\hline 6 & -1385.47 & 2828.939 & 0.395125 & 2857.939 & 1433.766 \\
\hline
\end{tabular}

\subsection{Determining the number of latent classes}

As discussed in Section 2, the measures of AIC, $\bar{\rho}^{2}$, AIC3, and BIC were applied to help determine the number of latent classes. We attempted various numbers of classes $(1,2,3,4$, 
5, and 6 classes) and summarize the statistics in Table 3. The log-likelihood values at convergence reveal that the greater the number of classes, the better the model's fit is. This is not surprising, because log-likelihood values normally increase in magnitude when there are more parameters to be estimated (Shen and Saijo, 2009). From the measures of AIC3 and $\mathrm{BIC}$, we find that the minimum values are in the 4-class model, suggesting that the 4-class model is optimal. Furthermore, although the minimum of AIC and the maximum of $\bar{\rho}^{2}$ seem to support the 6 -class model as the best solution, the improvement from 4 classes to 6 classes is so small as to be negligible. Therefore, we determined to select 4 classes for estimating the LCL models in this study.

\subsection{Results of the 4-class LCL Model}

The estimated results of the 4-class LCL model are listed in Table 4. For comparison purpose, the MNL estimates are also provided. Two alternative specific constants (i.e., Payment A and Payment B) and two attributes (i.e., Hourly pay and Pay for each snack sold) were estimated as explanatory variables. Note again that Payment $A$ and Payment $B$ represent the pure fixed payment and pure performance-related payment systems, respectively.

Table 4. Estimation Results of the MNL and LCL Models

\begin{tabular}{|l|l|l|l|l|l|}
\hline & \multirow{2}{*}{ MNL } & \multicolumn{5}{|c|}{ LCL } \\
\cline { 3 - 6 } & & Class 1 & Class 2 & Class 3 & Class 4 \\
\hline \hline Payment A & $-0.6545^{* *}$ & $7.9013^{* *}$ & -0.9915 & $-5.6060^{* * *}$ & -1.0971 \\
\hline Payment B & $0.6656^{*}$ & -1.0220 & 0.8821 & $2.6326^{* *}$ & $4.0328^{*}$ \\
\hline Hourly pay & $0.0089^{* * *}$ & $0.0129^{* * *}$ & $0.0213^{* * *}$ & $0.0206^{* * *}$ & $0.0116^{* * *}$ \\
\hline $\begin{array}{l}\text { Pay for each snack } \\
\text { sold }\end{array}$ & $0.0714^{* * *}$ & $0.1804^{* * *}$ & $0.1634^{* * *}$ & $0.1214^{* * *}$ & $0.0962^{* * *}$ \\
\cline { 1 - 3 } Class probability & & 0.1332 & 0.3057 & 0.3668 & 0.1943 \\
\hline Log likelihood & -2089.32 & -1396.46 & & & \\
\cline { 1 - 3 } Pseudo R ${ }^{2}$ & 0.1065 & 0.4066 & & & \\
\cline { 1 - 3 } Predictive power & $50.27 \%$ & $77.68 \%$ & & \\
\cline { 1 - 4 } Observations & 2142 & 2142 & & \\
\hline
\end{tabular}

Notes: Predictive power refers to the proportion of choices correctly predicted by the model. *, **, and ${ }^{* * *}$ denote that the estimated parameter is significantly different from zero at the $10 \%, 5 \%$, and $1 \%$ level, respectively. Standard errors and $z$ values are omitted to save space.

First, look at the parameters of Payment $A$ and Payment B. Compared to the MNL estimates, the LCL estimates help us achieve fresh insight into respondents' preferences over 
different types of payment systems. Based on the estimated signs and significances, about $37 \%$ of the respondents (i.e., respondents in class 3) prefer the pure performance-related payment system and do not prefer the pure fixed payment system, relative to the payment system that mixes both fixed pay and performance-related pay. In contrast, about $13 \%$ of the respondents (i.e., respondents in class 1) and 19\% of the respondents (i.e., respondents in class 4) favor the pure fixed payment and the pure performance-related payment systems, respectively, over the mixed payment system. Furthermore, the remaining $30 \%$ of the respondents (i.e., respondents in class 2) have no differences with respect to type of payment system.

Concerning the estimated positive parameters of Hourly pay and Pay for each snack sold, we find that both are highly significant in all classes. This result indicates that the higher the pay, either fixed or performance-related, the happier the respondents are. Furthermore, based on these estimated parameters, we can calculate the values of the marginal tradeoff between fixed pay and performance-related pay for each class by dividing the latter parameter by the former. The values are calculated as 13.98 in class 1, 7.67 in class 2, 5.89 in class 3, and 8.29 in class 4 . It should be noted that the larger the value of the tradeoff, the more likely respondents are to prefer the marginal increase in the fixed pay. Therefore, from these classbased values, it is clear that respondents in class 1 prefer the marginal increase in the fixed pay the most, and respondents in class 3 prefer the marginal increase in the fixed pay the least. It is worth noting that this evidence is consistent with that obtained from the specific alternatives. That is to say, on the one hand the respondents in class 1 prefer the pure fixed payment system to the other two, and thus they have the highest value of the marginal tradeoff; on the other hand the respondents in class 3 prefer the pure performance-related payment system to the other two, and thus they have the lowest value of the marginal tradeoff.

\subsection{Risk Preference and Individual Tradeoff Between Fixed Pay and Performance- Related Pay}

We asked the respondents to answer three questions relating to risk attitudes in the survey, aiming at eliciting their risk preferences. The detailed questions are provided in the Appendix. We categorized respondents' risk preferences into risk-averse, risk-neutral, and risk-loving by the criterion that risk-averse is assigned by Q1 if the answer of Q1 is greater than 0.5, by Q2 if the answer of Q2 is greater than 0.25, and by Q3 if the answer of Q3 is smaller than 0.5; risk-neutral is assigned by Q1 if the answer of Q1 is equal to 0.5, by Q2 if the answer of Q2 is equal to 0.25 , and by Q3 if the answer of Q3 is equal to 0.5; and risk-loving is assigned by Q1 if the answer of Q1 is smaller than 0.5, by Q2 if the answer of Q2 is smaller than 0.25, and by Q3 if the answer of Q3 is larger than 0.5. 
By using the results of the 4-class LCL model and conditioning these on the individual choices, it is possible to obtain the values of the marginal tradeoff between fixed pay and performance-related pay for each individual. The mean individual tradeoffs categorized into the above-described three risk categories are presented in Figure 1. As shown in the figure, the mean individual tradeoff between fixed pay and performance-related pay decreases as the degree of risk-seeking goes up for all three questions. This is plausible because risk lovers are usually willing to sacrifice assured pay in order to pursue a greater possible reward; as a result, they are willing to accept a smaller marginal increase in the performance-related pay. We also conducted a two-tailed $t$-test for the null hypothesis that there is no difference in the mean tradeoff between risk averters and risk lovers. As a result, the null hypothesis is significantly rejected at the 5\% level in Q1 $(t=2.225, p=0.027$, degree of freedom $=222)$ and 10\% level in Q2 $(t=1.783, p=0.076$, degree of freedom $=186)$ and Q3 $(t=1.764, p=$ 0.079 , degree of freedom $=180$ ), which provides supportive evidence that risk lovers are willing to accept a smaller ratio of performance-related pay to fixed pay.

Figure 1.

Estimated Individual Tradeoff Between Fixed Pay And Performance-Related Pay

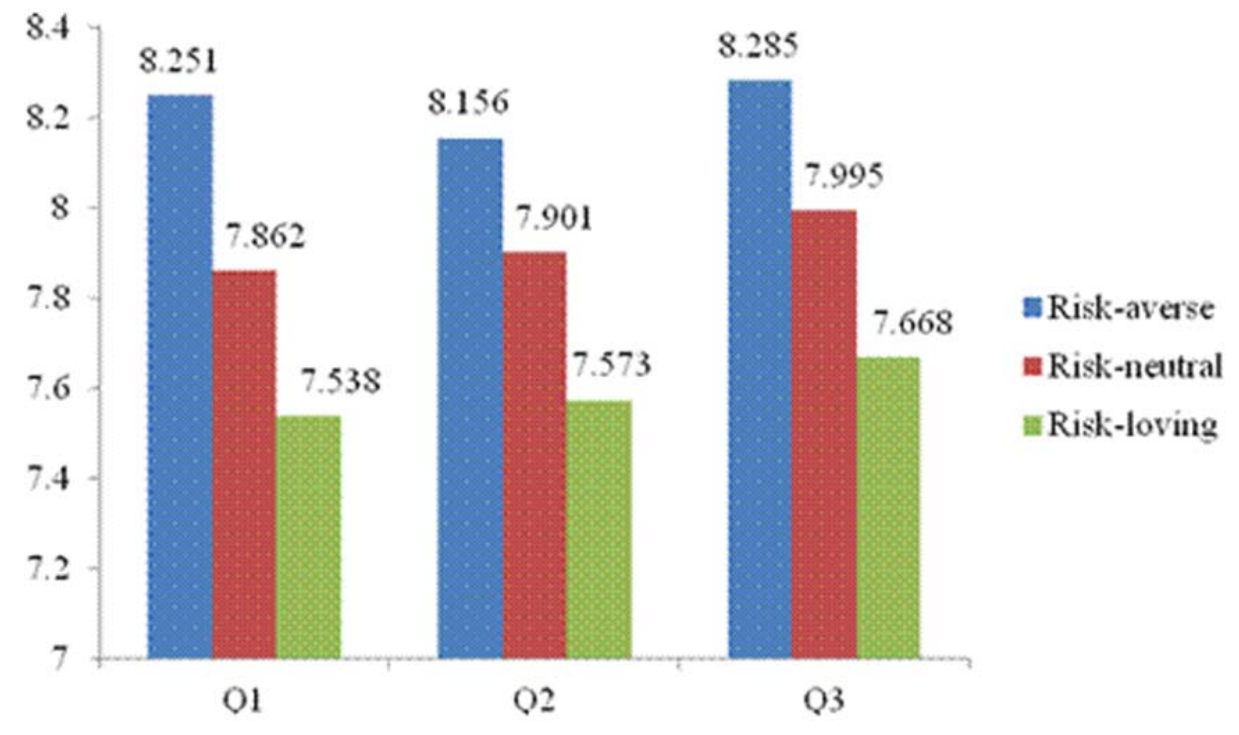

\section{Conclusions}

The latent class logit approach allows us to estimate both the values of the class-based and individual-based marginal tradeoffs between fixed pay and performance-related pay. Our empirical results imply that respondents belonging to different classes have not only different 
preferences with respect to the fixed payment and/or performance-related payment systems per se but also different values of the marginal tradeoff between fixed pay and performancerelated pay. In other words, individuals' preferences for the payment system and the magnitudes of tradeoffs between different kinds of pay are different. This raises an important implication that firms or employers should design more-flexible payment systems to meet with their employees' preferences for payment. However, this is very difficult to put into operation in the real world because firms or employers do not normally know their employees' true preferences in advance. Our analysis on linking the values of the tradeoff between fixed pay and performance-related pay with risk preferences may be, to some extent, a shortcut to solve this problem. Our result suggests that risk lovers are more willing to choose a performance-related payment system and risk averters are more willing to choose a fixed payment system. Based on this, individuals' preferences for payment systems can be elicited from their risk preferences, while eliciting risk preference is considered to be relatively easy.

\section{Appendix. Questions related to risk preferences}

Q1. There are two alternatives. Alternative 1 is that you will obtain 100 thousand JP yen with a probability of $100 \%$. Alternative 2 is that you will obtain 200 thousand JP yen with a probability of ( $\quad$ ). Please fill in the parenthesis with a numeral so that these two alternatives have the same value to you.

Q2. There are two alternatives. Alternative 1 is that you will obtain 100 thousand JP yen with a probability of $50 \%$. Alternative 2 is that you will obtain 200 thousand JP yen with a probability of ( ) \%. Please fill in the parenthesis with a numeral so that these two alternatives have the same value to you.

Q3. When you go out, you will bring the umbrella if a ( ) \% chance of rain is announced as the weather forecast. Please fill in the parenthesis with a numeral.

\section{References}

Baruch, Y., K. Wheeler, and X. Zhao (2004), Performance-related pay in Chinese professional sports. International Journal of Human Resource Management 15(1): 245259.

Bhat, C. R. and R. Gossen (2004), A mixed multinomial logit model analysis of weekend recreational episode type choice. Transportation Research Part B 38: 767-787.

Bjørner, T. B., L. G. Hansen, and C. R. Russell (2004), Environmental labeling and consumers' choice: An empirical analysis of the effect of the Nordic Swan. Journal of Environmental Economics and Management 47: 411-434. 
Review of Economic Analysis 6 (2014) 119-131

Booth, A. L. and J. Frank (1999), Earnings, productivity and performance related pay. Journal of Labor Economics 17(3): 447-464.

Boxall, P. C. and W. L. Adamowicz (2002), Understanding heterogeneous preferences in random utility models: A latent class approach. Environmental and Resource Economics 23: 421-446.

Green, C. and J. S. Heywood (2008), Does performance pay increase job satisfaction? Economica 75: 710-728.

Greene, W. H. and D. A. Hensher (2003), A latent class model for discrete choice analysis: contrast with mixed logit. Transportation Research Part B 37: 681-698.

Hess, S., M. Bierlaire, and J. W. Polak (2005), Estimation of value of travel-time savings using mixed logit models. Transportation Research Part A 39: 221-236.

Heywood, J. S. and X. Wei (2006), Performance pay and job satisfaction. Journal of Industrial Relations 48: 523-540.

Lazear, E. P (2000), Performance pay and productivity. American Economic Review 90(5): 1346-1391.

Louviere, J. J., D. A. Hensher, and J. D. Swait (2000), Stated Choice Methods: Analysis and Applications. Cambridge University Press.

McFadden, D. and K. Train (2000), Mixed MNL models for discrete response. Journal of Applied Econometrics 15: 447-470.

McNabb, R. and K. Whitfield (2007), The impact of varying types of performance-related pay and employee participation on earnings. International Journal of Human Resource Management 18(6): 1004-1025.

Morey, E., J. Thacher, and W. Breffle (2006), Using angler characteristics and attitudinal data to identify environmental preference classes: A latent-class model. Environmental and Resource Economics 34: 91-115.

Pearce, J. L. and J. L. Perry (1983), Federal merit pay: A longitudinal analysis. Public Administration Review 43(4): 315-325.

Perry, J. L., T. A. Engbers, and Y. J. So (2009), Back to the future? Performance-related pay, empirical research, and the perils of persistence. Public Administration Review 69(1): 3951.

Perry, J. L., D. Mesch, and L. Paarlberg (2006), Motivating employees in a new governance era: The performance paradigm revisited. Public Administration Review 66(4): 505-514.

Revelt, D. and K. Train (1998), Incentives for appliance efficiency in a competitive energy environment: Random parameters logit models of households' choices. Review of Economics and Statistics 80: 647-657.

Swait, J. (1994), A structural equation model of latent segmentation and product choice for cross-sectional revealed preference choice data. Journal of Retailing and Consumer Services 1: 77-89. 
Shen, J. (2006), A review of stated choice method. International Public Policy Research 10(2): 97-121.

Shen, J. and T. Saijo (2009), Does energy efficiency label alter consumers' purchasing decision? A latent class approach on Shanghai data. Journal of Environmental Management 90: 3561-3573.

Swait, J. (2007), Advanced choice models. In B. J. Kanninen (Ed), Valuing Environmental Amenities Using Stated Choice Studies. Springer.

Train, K. (1998), Recreation demand models with taste differences over people. Land Economics 74: 230-239.

Vroom, V. H. (1964), Work and Motivation. New York: Wiley. 